QUARTERLY OF APPLIED MATHEMATICS

VOLUME LXVII, NUMBER 3

SEPTEMBER 2009, PAGES 541-552

S 0033-569X(09)01138-3

Article electronically published on May 12, 2009

\title{
JUSTIFICATION OF A GALERKIN METHOD FOR A REGULARIZED CAUCHY SINGULAR INTEGRO-DIFFERENTIAL EQUATION
}

BY

\author{
A. I. FEDOTOV
}

Kazan State University, Research Institute of Mathematics and Mechanics, Kazan, 420008 Russia

This paper is dedicated to Vladimir Baicher and Joellen Jarvi.

Abstract. For one class of the singular integro-differential equations with Cauchy kernel on an interval, a Galerkin method is justified. The convergence is proved and the error estimation is given.

1. Introduction. In [1] a Galerkin method is proposed for the Cauchy-type singular linear integro-differential equation

$$
x^{\prime}(t)+\lambda \int_{-1}^{1} \frac{x(\tau) d \tau}{\tau-t}=g(t), \quad|t|<1
$$

subject to

$$
x(-1)=x(1)=0 .
$$

Here $x(\tau)$ is the desired unknown and $g(t)$ are given functions on the interval $[-1,1]$, $\lambda$ is a given real number and the singular integral is to be interpreted as the CauchyLebesgues principle value. Moreover, $x(\tau)$ are supposed to be even and $g(t)$ to be odd functions.

Received February 23, 2008.

2000 Mathematics Subject Classification. Primary 45L05; Secondary 45E05, 65R20.

Key words and phrases. Singular integro-differential equations, Galerkin method.

E-mail address: fedotov@mi.ru

Reverts to public domain 2009 Brown University 
The method proposed in [1] for solving the problem (1.1), (1.2) is based on the conversion of the original equation (1.1) to the equation

$$
\begin{gathered}
\frac{1}{\pi^{2} \sqrt{1-t^{2}}} \int_{-1}^{1} \frac{\sqrt{1-\tau^{2}} x^{\prime}(\tau) d \tau}{t-\tau}+\lambda x(t)-\frac{C}{\pi \sqrt{1-t^{2}}} \\
=\frac{1}{\pi \sqrt{1-t^{2}}} \int_{-1}^{1} \frac{\sqrt{1-\tau^{2}} g(\tau) d \tau}{t-\tau}, \quad|t|<1, \\
C=\int_{-1}^{1} x(\tau) d \tau,
\end{gathered}
$$

by inverting the singular integral in the equation (1.1) and then applying the Galerkin method to the equation (1.3). To do this, the author substitutes for the unknown function $x(\tau)$ in the equation (1.3) the polynomial

$$
x_{n}(\tau)=\sum_{k=1}^{n} a_{2 k}\left(T_{2 k}(\tau)-1\right)
$$

and then equates the first $n$ Fourier coefficients of the left- and right-hand sides of the obtained equation with respect to the system of Chebyshev polynomials of the first kind $\left\{T_{2 j}(t)\right\}_{j=0}^{n-1}$ with the weight-function $p(t)=\left(1-t^{2}\right)^{-1 / 2}$. The calculation scheme derived in this case is really "remarkably compact" [1. One can check it by comparing, for example, with the calculation scheme of the Galerkin method applied directly to the original equation (1.1) in 2.

However, the conversion of the equation (1.1) was made according to the general formula of inversion of the singular integrals 3 , which does not consider the condition (1.2). It is better to use here the particular case of the inversion formula for functions bounded at the ends of the interval $[-1,1]$ (see [4]). In this case an even more compact calculation scheme could be obtained.

In this paper we convert the original equation (1.1) according to the aforementioned particular formula, investigate the unique solvability of the obtained equation, construct the Galerkin method for this equation, prove its convergence and estimate the error of the approximate solution.

2. Some facts from the theory of the singular integral equations. Here we review some facts from the theory of the singular integral equations (see [4]).

Consider the singular integral equation of the first kind

$$
\int_{-1}^{1} \frac{x(\tau) d \tau}{\tau-t}=g(t), \quad|t|<1
$$

where $x(\tau)$ is the desired unknown and $g(t)$ are given functions on the interval $[-1,1]$. Suppose that $g(t)$ is Hölder continuous.

The function $Z(t)=(1-t)^{\gamma_{1}}(1+t)^{\gamma_{2}}$ is called the canonical function of the equation (2.1). Here

$$
\gamma_{1}=\lambda_{1}-\frac{1}{2}, \quad \gamma_{2}=\lambda_{2}+\frac{1}{2}
$$


and $\lambda_{1}, \lambda_{2}$ are integer numbers satisfying $\gamma_{1}, \gamma_{2} \in(-1,1)$. The number $\kappa=-\left(\lambda_{1}+\lambda_{2}\right)$ is called the index of the equation (2.1) and $\gamma_{1}, \gamma_{2}$ determine the class of its solutions.

It is easy to see that there are 4 pairs of possible values of $\gamma_{1}$ and $\gamma_{2}$. Correspondingly there are 4 pairs of values of $\lambda_{1}$ and $\lambda_{2}$ and 3 different values of $\kappa$ :

$\begin{array}{rrrrr}\lambda_{1} & \lambda_{2} & \gamma_{1} & \gamma_{2} & \kappa \\ 0 & -1 & -1 / 2 & -1 / 2 & 1 \\ 0 & 0 & -1 / 2 & 1 / 2 & 0 \\ 1 & -1 & 1 / 2 & -1 / 2 & 0 \\ 1 & 0 & 1 / 2 & 1 / 2 & -1\end{array}$

The functions

$$
p(t)=Z(t) \quad \text { and } \quad q(t)=Z^{-1}(t),
$$

non-negative and integrable on $[-1,1]$, determine the kind of exact solution of the equation (2.1) and the condition of its solvability in case of negative index.

For $\kappa=0$

or

$$
p(t)=\sqrt{\frac{1-t}{1+t}} \text { and } q(t)=\sqrt{\frac{1+t}{1-t}}
$$

$$
p(t)=\sqrt{\frac{1+t}{1-t}} \text { and } q(t)=\sqrt{\frac{1-t}{1+t}} .
$$

In this case the equation (2.1) is always uniquely solvable for any Hölder continuous right-hand side $g(t)$. The solution has the form

$$
x(t)=-\frac{p(t)}{\pi^{2}} \int_{-1}^{1} \frac{q(\tau) g(\tau) d \tau}{\tau-t}
$$

and is unbounded at $t=-1$ and bounded at $t=1$ for the first case of $p(t)$ and, on the other hand, is unbounded at $t=1$ and bounded at $t=-1$ for the second case of $p(t)$.

For $\kappa=1$,

$$
p(t)=\frac{1}{\sqrt{1-t^{2}}} \quad \text { and } \quad q(t)=\sqrt{1-t^{2}} ;
$$

the equation (2.1) is also solvable for any Hölder continuous right-hand side $g(t)$. The solution has the form

$$
x(t)=-\frac{p(t)}{\pi^{2}}\left(\int_{-1}^{1} \frac{q(\tau) g(\tau) d \tau}{\tau-t}-C \pi\right)
$$

and is not unique. It depends on the arbitrary constant $C$. Integrating (2.2), one can easily see that

$$
C=\int_{-1}^{1} x(\tau) d \tau
$$

This case is the most general one. The formula (2.2) contains all possible solutions of the equation (2.1).

For $\kappa=-1$,

$$
p(t)=\sqrt{1-t^{2}} \quad \text { and } \quad q(t)=\frac{1}{\sqrt{1-t^{2}}}
$$


the equation (2.1) is solvable only if $g(t)$ satisfies the condition

$$
\int_{-1}^{1} q(\tau) g(\tau) d \tau=0
$$

and has the form

$$
x(t)=-\frac{p(t)}{\pi^{2}} \int_{-1}^{1} \frac{q(\tau) g(\tau) d \tau}{\tau-y} .
$$

In this case $x(t)$ is bounded at the endpoints of the interval $[-1,1]$. So this case seems to be more suitable for the problem (1.1), (1.2) than the general case (2.2) used in 1 .

3. Auxiliary results. We cite here two well-known lemmas needed for the analysis of the unique solvability of the regularized equation and for the proof of the covergence of the method. See [5] for the first lemma and [6] for the second one.

Lemma 3.1. Let $A$ and $B$ be linear bounded operators mapping Banach space $X$ to Banach space $Y$. Let $A$ be invertible and $\|B\|\left\|A^{-1}\right\|<1$. Then the operator $A+B$ : $X \rightarrow Y$ is invertible also and

$$
\left\|(A+B)^{-1}\right\| \leq \frac{\left\|A^{-1}\right\|}{1-\|B\|\left\|A^{-1}\right\|} .
$$

Let $X, Y$ be Banach spaces and let $X_{n} \subset X, Y_{n} \subset Y, n=1,2, \ldots$, be their subspaces. Consider equations

$$
\begin{aligned}
K x=y, & K: X \rightarrow Y, \\
K_{n} x_{n}=y_{n}, & K_{n}: X_{n} \rightarrow Y_{n},
\end{aligned}
$$

where $K$ and $K_{n}$ are linear bounded operators.

Lemma 3.2. Let $K: X \rightarrow Y$ be invertible and

$$
\left\|K-K_{n}\right\|_{X_{n} \rightarrow Y} \rightarrow 0 \text { as } n \rightarrow \infty .
$$

Then for $n$ satisfying

$$
q_{n}=\left\|K^{-1}\right\|\left\|K-K_{n}\right\|<1, \quad K-K_{n}: X_{n} \rightarrow Y,
$$

an approximate equation (3.2) has the unique solution $x_{n}^{*} \in X_{n}$ for any right-hand side $y_{n} \in Y_{n}$ and the estimation

$$
\left\|x^{*}-x_{n}^{*}\right\| \leq \frac{\left\|K^{-1}\right\|}{1-q_{n}}\left(\left\|y-y_{n}\right\|+q_{n}\|y\|\right)
$$

is valid, where $x^{*}=K^{-1} y$ is the exact solution of the equation (3.1). 
4. Preliminary evaluations and solvability analysis. Let us rewrite the equation (1.1) as

$$
\int_{-1}^{1} \frac{\lambda x(\tau) d \tau}{\tau-t}=g(t)-x^{\prime}(t)
$$

and invert the singular integral according to (2.5). We will have the equation

$$
\lambda x(t)=-\frac{\sqrt{1-t^{2}}}{\pi^{2}} \int_{-1}^{1} \frac{g(\tau)-x^{\prime}(\tau)}{\sqrt{1-\tau^{2}}(\tau-t)} d \tau
$$

and the solvability condition (2.4) will take the form

$$
\int_{-1}^{1} \frac{g(\tau)-x^{\prime}(\tau)}{\sqrt{1-\tau^{2}}} d \tau=0
$$

It is easy to check that the derivative $x^{\prime}(t)$ of the even function $x(t)$ is the odd function. As the function $g(t)$ is an odd function too, the condition (4.3) is obviously fulfilled.

Now we rewrite the equation (4.2) as

$$
\frac{1}{\pi} \int_{-1}^{1} \frac{x^{\prime}(\tau) d \tau}{\sqrt{1-\tau^{2}}(\tau-t)}-\frac{\pi \lambda x(t)}{\sqrt{1-t^{2}}}=\frac{1}{\pi} \int_{-1}^{1} \frac{g(\tau) d \tau}{\sqrt{1-\tau^{2}}(\tau-t)}
$$

and consider it as an operator equation

$$
K x \equiv G x+V x=y, \quad K: X \rightarrow Y .
$$

Here

$$
X=\left\{x \in W_{2, p}^{1} \mid x(-t)=x(t), t \in[-1,1], x(-1)=x(1)=0\right\}
$$

is the space of all even functions vanishing at the endpoints $t=-1, t=1$ of the interval, whose first derivative is square integrable on $[-1,1]$ with the weight-function $p(t)=\left(1-t^{2}\right)^{-1 / 2}$, and

$$
Y=\left\{y \in L_{2, q} \mid y(-t)=y(t), t \in[-1,1]\right\}
$$

is the space of all even functions square integrable on $[-1,1]$ with the weight-function $q(t)=\left(1-t^{2}\right)^{1 / 2}$. In the space $X$ define the inner product

$$
\langle x, y\rangle_{X}=\int_{-1}^{1} x^{\prime}(\tau) y^{\prime}(\tau) p(\tau) d \tau, \quad x, y \in X,
$$

and the norm

$$
\|x\|_{X}=\sqrt{\langle x, x\rangle_{X}}, \quad x \in X .
$$

In the space $Y$ define correspondingly the inner product

$$
\langle x, y\rangle_{Y}=\int_{-1}^{1} x(\tau) y(\tau) q(\tau) d \tau, \quad x, y \in Y,
$$

and the norm

$$
\|y\|_{Y}=\sqrt{\langle y, y\rangle}, \quad y \in Y .
$$

With these norms the spaces $X$ and $Y$ become Banach spaces. In addition we will need the space

$$
Z=\left\{g \in L_{2, p} \mid g(-t)=-g(t), \quad t \in[-1,1]\right\}
$$


of all odd functions square integrable on $[-1,1]$ with the weight-function $p(t)=$ $\left(1-t^{2}\right)^{-1 / 2}$. In this space we define the inner product

$$
\langle g, h\rangle_{Z}=\int_{-1}^{1} g(\tau) h(\tau) p(\tau) d \tau, \quad g, h \in Z,
$$

and the norm

$$
\|g\|_{Z}=\sqrt{\langle g, g\rangle_{Z}}, \quad g \in Z
$$

In the equation (4.5)

$$
\begin{gathered}
G=S D, \quad D x=x^{\prime}, \quad(S x)(t)=\frac{1}{\pi} \int_{-1}^{1} \frac{x(\tau) d \tau}{\sqrt{1-\tau^{2}}(\tau-t)}, \\
(V x)(t)=-\frac{\pi \lambda x(t)}{\sqrt{1-t^{2}}}, \quad y(t)=(S g)(t) .
\end{gathered}
$$

Define now the sequence of polynomials $\left\{\Phi_{2 k}(t)\right\}_{k=1}^{\infty}$

$$
\begin{gathered}
\Phi_{2}(t)=\frac{T_{2}(t)-1}{4}, \\
\Phi_{2 k}(t)=\frac{1}{4}\left(\frac{T_{2 k}(t)-1}{k}-\frac{T_{2 k-2}(t)-1}{k-1}\right), \quad k=2,3, \ldots,
\end{gathered}
$$

where $\left\{T_{2 k}(t)\right\}_{k=1}^{\infty}$ are even Chebyshev polynomials of the first kind. As

$$
\Phi_{2 k}^{\prime}(t)=T_{2 k-1}(t), \quad \Phi_{2 k}(-1)=\Phi_{2 k}(1)=0, \quad k=1,2, \ldots,
$$

then $\left\{\Phi_{2 k}(t)\right\}_{k=1}^{\infty}$ is a full system of orthogonal polynomials in $X$. In $Y$ the full system of orthogonal polynomials is the system of even Chebyshev polynomials of the second kind $\left\{U_{2 k}(t)\right\}_{k=0}^{\infty}$ and in $Z$ the same system is the system of odd Chebyshev polynomials of the first kind $\left\{T_{2 k-1}(t)\right\}_{k=1}^{\infty}$.

Theorem 4.1. For any real $\lambda,|\lambda| \leq \frac{4}{\pi \sqrt{\pi^{2}-4}}$, and any $g \in Z$ the problem (4.4), (1.2) has the unique solution $x^{*}=K^{-1} y \in X$ and the estimation

$$
\left\|K^{-1}\right\|_{Y \rightarrow X} \leq \frac{4}{4-\pi|\lambda| \sqrt{\pi^{2}-4}}
$$

is valid.

Proof. Let us estimate the norm of the operator $G: X \rightarrow Y$. To do this, take an arbitrary $x \in X$ and expand it by its Fourier series with respect to $\left\{\Phi_{2 k}(t)\right\}_{k=1}^{\infty}$, i.e.,

$$
\begin{gathered}
x(t)=\sum_{k=1}^{\infty} \hat{x}(2 k) \Phi_{2 k}(t), \\
\hat{x}(2 k)=\frac{2}{\pi} \int_{-1}^{1} x^{\prime}(\tau) \Phi_{2 k}^{\prime}(\tau) p(\tau) d \tau, \quad k=1,2, \ldots
\end{gathered}
$$

The norm of $x(t)$ in $X$ is equal to

$$
\|x\|_{X}=\left(\frac{\pi}{2} \sum_{k=1}^{\infty} \hat{x}^{2}(2 k)\right)^{1 / 2} .
$$


Applying the operator $G$ to $x(t)$, we will have

$$
\begin{gathered}
(G x)(t)=\frac{1}{\pi} \int_{-1}^{1} \frac{x^{\prime}(\tau) d \tau}{\sqrt{1-\tau^{2}}(\tau-t)} \\
=\frac{1}{\pi} \sum_{k=1}^{\infty} \hat{x}(2 k) \int_{-1}^{1} \frac{T_{2 k-1}(\tau) d \tau}{\sqrt{1-\tau^{2}}(\tau-t)}=\sum_{k=1}^{\infty} \hat{x}(2 k) U_{2 k-2}(t) .
\end{gathered}
$$

The norm of $G x$ in $Y$ is

$$
\|G x\|_{Y}=\left(\frac{\pi}{2} \sum_{k=1}^{\infty} \hat{x}^{2}(2 k)\right)^{1 / 2}=\|x\|_{X} .
$$

This means that $\|G\|_{X \rightarrow Y}=1$.

Let us show now that the operator $G$ is invertible. Take arbitrary $y \in Y$ and expand it by its Fourier series with respect to polynomials $\left\{U_{2 k-2}(t)\right\}_{k=1}^{\infty}$, i.e.,

$$
\begin{gathered}
y(t)=\sum_{k=1}^{\infty} \hat{y}(2 k) U_{2 k-2}(t), \\
\hat{y}(2 k)=\frac{2}{\pi} \int_{-1}^{1} y(\tau) U_{2 k-2}(\tau) q(\tau) d \tau, \quad k=1,2, \ldots
\end{gathered}
$$

From (4.6) it is easy to see that

$$
\left(G^{-1} y\right)(t)=x(t)=\sum_{k=1}^{\infty} \hat{y}(2 k) \Phi_{2 k}(t)
$$

and thus the operator $G: X \rightarrow Y$ is invertible. Moreover, as

$$
\left\|G^{-1} y\right\|_{X}=\left(\frac{\pi}{2} \sum_{k=1}^{\infty} \hat{y}^{2}(2 k)\right)^{1 / 2}=\|y\|_{Y},
$$

then $\left\|G^{-1}\right\|_{Y \rightarrow X}=1$.

Now, to demonstrate $K=G+V$ has an inverse, it is sufficient, according to Lemma 3.1, to show that $\|V\|_{X \rightarrow Y}<1$. Let us, using once more the arbitrary element $x \in X$, estimate the norm of the operator $V: X \rightarrow Y$. According to the Cauchy-Bunyakovskii inequality

$$
\begin{aligned}
& \|V x\|_{Y}^{2}=\pi^{2} \lambda^{2} \int_{-1}^{1} \frac{x^{2}(\tau) d \tau}{\sqrt{1-\tau^{2}}}=\pi^{2} \lambda^{2} \int_{-1}^{1} \frac{1}{\sqrt{1-\tau^{2}}}\left(\sum_{k=1}^{\infty} \hat{x}^{2}(2 k) \Phi_{2 k}(\tau)\right)^{2} d \tau \\
& \leq \pi^{2} \lambda^{2} \sum_{k=j}^{\infty} \hat{x}^{2}(2 j) \int_{-1}^{1} \frac{1}{\sqrt{1-\tau^{2}}} \sum_{k=1}^{\infty} \Phi_{2 k}^{2}(\tau) d \tau=2 \pi \lambda^{2}\|x\|_{X}^{2} \sum_{k=1}^{\infty} \int_{-1}^{1} \frac{\Phi_{2 k}^{2}(\tau) d \tau}{\sqrt{1-\tau^{2}}}
\end{aligned}
$$

The integrals in the last expression could be calculated explicitly, i.e.,

$$
\begin{gathered}
\int_{-1}^{1} \frac{\Phi_{2}^{2}(\tau) d \tau}{\sqrt{1-\tau^{2}}}=\frac{3 \pi}{32} \\
\int_{-1}^{1} \frac{\Phi_{2 k}^{2} d \tau}{\sqrt{1-\tau^{2}}}=\frac{\pi\left(2 k^{2}-2 k+3\right)}{32 k^{2}(k-1)^{2}}, \quad k=2,3, \ldots
\end{gathered}
$$


So

$$
\|V x\|_{Y}^{2} \leq \frac{\pi^{2} \lambda^{2}}{16}\|x\|_{X}^{2}\left(3+\sum_{k=2}^{\infty} \frac{2 k^{2}-2 k+3}{k^{2}(k-1)^{2}}\right)=\frac{\pi^{2} \lambda^{2}}{16}\|x\|_{X}^{2}\left(\pi^{2}-4\right),
$$

and the condition $\|V\|_{Y \rightarrow X}<1$ will be valid for all $\lambda, \quad|\lambda|<\frac{4}{\pi \sqrt{\pi^{2}-4}}$. For such $\lambda$ 's, according to Lemma 3.1, the inverse $K^{-1}: Y \rightarrow X$ exists and its norm could be estimated:

$$
\left\|K^{-1}\right\|_{Y \rightarrow X} \leq \frac{4}{4-\pi|\lambda| \sqrt{\pi^{2}-4}}
$$

Theorem 4.1 is proved.

5. Calculation scheme. We will seek the approximate solution of the equation (4.4) as a polynomial

$$
x_{2 n}(\tau)=\sum_{k=1}^{n} a_{2 k} \Phi_{2 k}(\tau)
$$

with the unknown coefficients $\left\{a_{2 k}\right\}_{k=1}^{n}$. Substituting $x_{2 k}(\tau)$ in the left-hand side of the equation (4.4), we will have

$$
\begin{gathered}
\left(G x_{2 n}\right)(t)=\sum_{k=1}^{n} a_{2 k} U_{2 k-2}(t), \\
\left(V x_{2 n}\right)(t)=-\frac{\pi \lambda}{\sqrt{1-t^{2}}} \sum_{k=1}^{n} a_{2 k} \Phi_{2 k}(t), \\
\left(K x_{2 n}\right)(t)=\sum_{k=1}^{n} a_{2 k} U_{2 k-2}(t)-\frac{\pi \lambda}{\sqrt{1-t^{2}}} \sum_{k=1}^{n} a_{2 k} \Phi_{2 k}(t) .
\end{gathered}
$$

We will first expand the function $g \in Z$ by its Fourier series with respect to the polynomials $\left\{T_{2 k-1}(t)\right\}_{k=0}^{\infty}$, i.e.,

$$
\begin{gathered}
g(t)=\sum_{k=1}^{\infty} \hat{g}(2 k-1) T_{2 k-1}(t), \\
\hat{g}(2 k-1)=\frac{2}{\pi} \int_{-1}^{1} g(\tau) T_{2 k-1}(\tau) p(\tau) d \tau, \quad k=1,2, \ldots,
\end{gathered}
$$

and then apply the operator $S$ to it:

$$
(S g)(t)=\sum_{k=1}^{\infty} \hat{g}(2 k-1) U_{2 k-2}(t)
$$


The Fourier coefficients of the left-hand side $\left(K x_{2 n}\right)(t)$ and right-hand side $(S g)(t)$ of the equation (4.4) with respect to the Chebyshev polynomials of the second kind $\left\{U_{2 j-2}(t)\right\}_{j=1}^{\infty}$ are

$$
\begin{gathered}
\left(\widehat{K x_{2 n}}\right)(2 j-2)=\left(\widehat{G x_{2 n}}\right)(2 j-2)+\left(\widehat{V x_{2 n}}\right)(2 j-2), \quad j=1,2, \ldots, \infty, \\
\left(\widehat{G x_{2 n}}\right)(2 j-2)=a_{2 j}, \quad j=1,2, \ldots, n, \\
\left(\widehat{V x_{2 n}}\right)(2 j-2)=\frac{2}{\pi} \int_{-1}^{1}\left(V x_{2 n}\right)(\tau) U_{2 j-2}(\tau) q(\tau) d \tau \\
=-2 \lambda \sum_{k=1}^{n} a_{2 k} \int_{-1}^{1} \Phi_{2 k}(\tau) U_{2 j-2}(\tau) d \tau \\
=-\frac{2 \lambda}{2 j-1} \sum_{k=1}^{n} a_{2 k}\left(\frac{1}{(2 j+2 k-2)^{2}-1}+\frac{1}{(2 j-2 k)^{2}-1}\right), \quad j=1,2 \ldots, \infty, \\
(\widehat{S g})(2 j-2)=\hat{g}(2 j-1), \quad j=1,2, \ldots, \infty .
\end{gathered}
$$

Equating the first $n$ coefficients of the left- and right-hand sides, we will have the system of the linear algebraic equations

$$
\begin{gathered}
a_{2 j}-\frac{2 \lambda}{2 j-1} \sum_{k=1}^{n} a_{2 k}\left(\frac{1}{(2 j+2 k-2)^{2}-1}+\frac{1}{(2 j-2 k)^{2}-1}\right) \\
=\hat{g}(2 j-1), \quad j=1,2, \ldots, n,
\end{gathered}
$$

of the Galerkin method for the equation (1.1).

\section{Justification of the method.}

Theorem 6.1. Let $g$ in the right-hand side of equation (1.1) belong to $Z$ and let $|\lambda|<$ $\frac{4}{\pi \sqrt{\pi^{2}-4}}$. Then for all natural numbers $n$ satisfying

$$
q_{n}=\frac{4|\lambda|}{\left(4-\pi|\lambda| \sqrt{\pi^{2}-4}\right)(2 n+1)}<1,
$$

the system of equations (5.3) has unique solution $\left\{a_{2 k}^{*}\right\}_{k=1}^{n}$ and approximate solutions

$$
x_{2 n}^{*}(\tau)=\sum_{k=1}^{n} a_{2 k}^{*} \Phi_{2 k}(\tau)
$$

converge to the exact solution $x^{*} \in X$ of the equation (1.1) with error estimation

$$
\left\|x^{*}-x_{2 n}^{*}\right\|_{X} \leq \frac{4}{\left(4-\pi|\lambda| \sqrt{\pi^{2}-4}\right)\left(1-q_{n}\right)}\left(E_{2 n-1}(g)_{Z}+q_{n}\|g\|_{Z}\right),
$$

where $E_{2 n-1}(g)_{Z}$ is the best approximation of the function $g \in Z$ by the polynomials of order not higher than $2 n-1$. 
Proof. Let $\mathcal{P}_{2 n}$ denote an operator which assigns the Fourier sum

$$
\begin{gathered}
\left(\mathcal{P}_{2 n} y\right)(\tau)=\sum_{j=1}^{n} \hat{y}(2 j-2) U_{2 j-2}(\tau), \\
\hat{y}(2 j-2)=\frac{2}{\pi} \int_{-1}^{1} y(\tau) U_{2 j-2}(\tau) q(\tau) d \tau, \quad j=1,2, \ldots, n,
\end{gathered}
$$

to each function $y \in Y$.

Let $X_{2 n}$ be the set of all even algebraic polynomials of order not higher than $2 n$ and let $Y_{2 n}=\mathcal{P}_{2 n} Y$. Then the system of equations (5.3) is equivalent to the operator equation

$$
K_{2 n} x_{2 n} \equiv \mathcal{P}_{2 n} K x_{2 n}=\mathcal{P}_{2 n} S g, \quad K_{2 n}: X_{2 n} \rightarrow Y_{2 n} .
$$

Equivalence here means that if the polynomial $x_{2 n}^{*}(\tau)$ is the solution of the equation (6.1), then the coefficients $\left\{a_{2 k}^{*}\right\}_{k=1}^{n}$ of its expansion

$$
x_{2 n}^{*}(\tau)=\sum_{k=1}^{n} a_{2 k}^{*} \Phi_{2 k}(\tau)
$$

are the solution of the system of the equations (5.3). So to prove the solvability of the system of the equations (5.3), it is sufficient to prove the solvability of the equation (6.1).

Let us show that the operators $K$ and $K_{2 n}$ are close to each other on $X_{n}$. For the arbitrary

$$
x_{2 n}(\tau)=\sum_{k=1}^{n} \hat{x}(2 k) \Phi_{2 k}(\tau) \in X_{2 n}
$$

we estimate the difference $K x_{2 n}-K_{2 n} x_{2 n}$ in $Y$. As

$$
\left(\mathcal{P}_{2 n} G x_{2 n}\right)(t)=\left(G x_{2 n}\right)(t),
$$

then

$$
K x_{2 n}-K_{2 n} x_{2 n}=V x_{2 n}-\mathcal{P}_{2 n} V x_{2 n},
$$

so

$$
\begin{gathered}
\left\|K x_{2 n}-K_{2 n} x_{2 n}\right\|_{Y}^{2}=\left\|V x_{2 n}-\mathcal{P}_{2 n} V x_{2 n}\right\|_{Y}^{2} \\
=\pi^{2} \lambda^{2} \int_{-1}^{1}\left(\sum_{k=1}^{n} \hat{x}_{2 n}(2 k)\left(\Phi_{2 k}(\tau) p(\tau)-\left(\mathcal{P}_{2 n}\left(\Phi_{2 k} p\right)\right)(\tau)\right)\right)^{2} q(\tau) d \tau \\
\leq \pi^{2} \lambda^{2} \sum_{k=1}^{n} \hat{x}_{2 n}^{2}(2 k) \sum_{k=1}^{n} \int_{-1}^{1}\left(\sum_{j=n}^{\infty} \hat{W}_{2 k}(2 j) U_{2 j}(\tau)\right)^{2} q(\tau) d \tau \\
=\pi^{2} \lambda^{2}\left\|x_{2 n}\right\|_{X}^{2} \sum_{k=1}^{n} \sum_{j=n}^{\infty} \hat{W}_{2 k}^{2}(2 j),
\end{gathered}
$$


where

$$
\begin{gathered}
W_{2 k}(\tau)=\Phi_{2 k}(\tau) p(\tau), \quad \hat{W}_{2 k}(2 j)=\frac{2}{\pi} \int_{-1}^{1} W_{2 k}(\tau) U_{2 j}(\tau) q(\tau) d \tau \\
=\frac{2}{\pi(2 j+1)}\left(\frac{1}{(2 j+2 k)^{2}-1}+\frac{1}{(2 j-2 k+2)^{2}-1}\right) \\
k=1,2, \ldots, n, \quad j=n, n+1, \ldots \infty .
\end{gathered}
$$

Once more using the Cauchy-Bunyakovskii inequality, we will estimate the double sum

$$
\begin{gathered}
\sum_{k=1}^{n} \sum_{j=n}^{\infty} \hat{W}_{2 k}^{2}(2 j)=\frac{4}{\pi^{2}} \sum_{k=1}^{n} \sum_{j=n}^{\infty} \frac{1}{(2 j+1)^{2}}\left(\frac{1}{(2 j+2 k)^{2}-1}+\frac{1}{(2 j-2 k+2)^{2}-1}\right)^{2} \\
\leq \frac{8}{\pi^{2}(2 n+1)^{2}} \sum_{j=n}^{\infty}\left(\sum_{k=1}^{n}\left(\frac{1}{(2 j+2 k)^{2}-1}\right)^{2}+\sum_{k=1}^{n}\left(\frac{1}{(2 j-2 k+2)^{2}-1}\right)^{2}\right. \\
=\frac{8}{\pi^{2}(2 n+1)^{2}} \sum_{j=0}^{\infty} \sum_{k=1}^{2 n} \frac{1}{\left(4(j+k)^{2}-1\right)^{2}} \leq \frac{8}{\pi^{2}(2 n+1)^{2}} \sum_{j=0}^{\infty} \sum_{k=1}^{\infty} \frac{1}{\left(4(j+k)^{2}-1\right)^{2}} \\
=\frac{8}{\pi^{2}(2 n+1)^{2}} \sum_{l=1}^{\infty} \frac{l}{\left(4 l^{2}-1\right)^{2}}=\frac{1}{\pi^{2}(2 n+1)^{2}} .
\end{gathered}
$$

Finally we have

$$
\begin{gathered}
\left\|K x_{2 n}-K_{2 n} x_{2 n}\right\|_{Y}^{2} \leq \frac{\lambda^{2}}{(2 n+1)^{2}}\left\|x_{2 n}\right\|_{X}^{2}, \\
\left\|K-K_{2 n}\right\|_{X \rightarrow Y} \leq \frac{|\lambda|}{2 n+1} .
\end{gathered}
$$

Now, according to Lemma 3.2, we can conclude that for all natural numbers $n$ satisfying

$$
q_{n}=\frac{4|\lambda|}{\left(4-\pi|\lambda| \sqrt{\pi^{2}-4}\right)(2 n+1)}<1,
$$

the operator equation (6.1) has the unique solution

$$
x_{2 n}^{*}(\tau)=\sum_{k=1}^{n} a_{2 k}^{*} \Phi_{2 k}(\tau),
$$

where the coefficients $\left\{a_{2 k}^{*}\right\}_{k=1}^{n}$ are the solution of the system of the equations (5.3), and the estimation

$$
\left\|x^{*}-x_{2 n}^{*}\right\|_{X} \leq \frac{4}{\left(4-\pi|\lambda| \sqrt{\pi^{2}-4}\right)\left(1-q_{n}\right)}\left(\left\|S g-\mathcal{P}_{2 n}(S g)\right\|_{Y}+q_{n}\|S g\|_{Y}\right)
$$

is valid.

The norm of the function $S g$ in $Y$ is equal to the norm of the function $g$ in $Z$, i.e.,

$$
\|S g\|_{Y}^{2}=\|g\|_{Z}^{2}=\frac{\pi}{2} \sum_{k=1}^{\infty} \hat{g}^{2}(2 k-1),
$$


the best approximation of the function $S g$ in $Y$ is equal to the best approximation of the function $g$ in $Z$, i.e.,

$$
\left\|S g-\mathcal{P}_{2 n} S g\right\|_{Y}^{2}=\frac{\pi}{2} \sum_{k=n+1}^{\infty} \hat{g}^{2}(2 k-1)=E_{2 n-1}^{2}(g)_{Z},
$$

and thus the estimation (6.2) can be rewritten as

$$
\left\|x^{*}-x_{2 n}^{*}\right\|_{X} \leq \frac{4}{\left(4-\pi|\lambda| \sqrt{\pi^{2}-4}\right)\left(1-q_{n}\right)}\left(E_{2 n-1}(g)_{Z}+q_{n}\|g\|_{Z}\right) .
$$

Theorem 6.1 is proved.

\section{REFERENCES}

[1] J. I. Frankel, A Galerkin solution to a regularized Cauchy singular integro-differential equation, Quart. Appl. Math. 2, 245-258 (1995). MR1330651(96b:45002)

[2] A. A. Badr, Integro-differential equation with Cauchy kernel, J. Comput. Appl. Math. 134, 191-199 (2001). MR1852565 (2002e:65199)

[3] A. S. Peters, A note on the integral equation of the first kind with a Cauchy kernel, Comm. Pure Appl. Math. 21, 57-61 (1963). MR0147859(26:5372)

[4] F. D. Gakhov, Boundary Value Problems, Pergamon Press, Oxford, 1966. MR0198152 (33:6311)

[5] M. A. Krasnosel'skii, G. M. Vainikko, P. P. Zabreiko, Ya. B. Rutitskii, V. Ya. Stetsenko, Approximate solution of operator equations, Noordhoff, Groningen, 1972. MR0385655 (52:6515)

[6] B. G. Gabdulkhaev, Optimal approximations of solutions of linear problems, Kazan State University, Kazan, 1980 (in Russian). MR630089 (83k:65047) 